\title{
Social Factors of Postpartum Depression Among Chinese Women
}

\author{
Linyan Li* \\ Department of Traditional Chinese Medicine, Wujing community health center, Shanghai University of Traditional Chinese \\ Medicine, China
}

\begin{abstract}
Objective: This article explores the various social factors contributing to postpartum depression among Chinese women by reviewing the relevant literature.

Methods: A comprehensive search was conducted by using multiple English and Chinese databases. Conclusion: A variety of social factors contribute to the development of postpartum depression among Chinese women. These factors include socio-cultural factors and socio-demographic factors. Chinese socio-cultural factors include son preference, the traditional post-childbirth custom of "doing the month," and the conflict between postpartum women and their mothers-in-law. Socio-demographic factors include socio-economic factors, education, ethnicity, and social support. Social support, especially family support, is closely associated with postpartum depression. Lack of social support is a high-risk factor for depression; strong social support is a preventive and protective factor for postpartum women.
\end{abstract}

Contribution to the Field: This article provides a detailed review of the social factors of postpartum depression among Chinese women. On the one hand, we find that a variety of unique socio-cultural factors are associated with postpartum depression, including son preference, the traditional post-childbirth custom of "doing the month," and the conflict between postpartum women and their mothers-in-law in Chinese culture. On the other hand, in China, socio-demographic factors, such as income, education, and ethnicity, are also considered risk factors for postpartum depression. Finally, social support, especially family support, is closely associated with postpartum depression. These findings contribute to the identification, prevention, and treatment of postpartum depression among Chinese women. The risk factors of postpartum depression should be considered in health planning and policymaking in Chinese society.

KEYWORDS: Postpartum depression; Social factors; Chinese women; Social support; Literature review

\section{INTRODUCTION}

Postpartum depression or postnatal depression is defined as a depressive disorder with an onset within four weeks after delivery, with symptoms including tearfulness, lack of interest or pleasure, fatigue, changes in sleep pattern, appetite or weight loss, feelings of worthlessness, and suicidal ideation and behaviors Diagnostic and Statistical Manual of Mental Disorders 2013. Based on a meta-analysis involving 291 studies from 56 countries, the global incidence of postpartum depression is approximately $17.7 \%$ Hahn-Holbrook [1]. The prevalence of postpartum depression in developing countries is roughly $20 \%$, World Health Organization [2], much higher than that in high-income countries such as the United States (11-20\%); Wisner et al. [3]. The reported prevalence of postpartum depression in China ranges from $11.38 \%$ to $34.00 \%$
Quick Response Code:

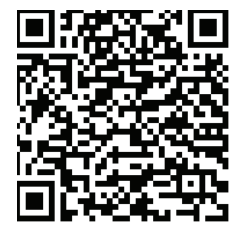

Address for correspondence: Linyan Li, Department of Traditional Chinese Medicine, Wujing community health center, Shanghai University of Traditional Chinese Medicine, China

Received: July 20, $2021 \quad$ Published: July 29, 2021

How to cite this article: Linyan Li. Social Factors of Postpartum Depression Among Chinese Women. 2021- 3(4) OAJBS.ID.000311. DOI: 10.38125/OAJBS.000311 
due to the various time frames, diagnostic criteria, screening scales, assessment methods, and cultural backgrounds in different studies Schardosim [4]; Zhang et al. [5]; Xiong [6]. As a global public health concern, postpartum depression has long-lasting devastating effects on the wellbeing of women and their family members. It not only affects the women's health and the marriage Ludermir et al. [7] but also impacts the cognitive and behavioral development of the offspring Closa-Monasterolo et al. [8]; Faleschini et al. [9]; Koutra et al. [10]. More concerning, postpartum depression contributes to serious consequences, such as self-harm, suicide, and infanticide Saralee et al. [11].

Given its high prevalence and severe consequences, it is important to identify the risk factors of postpartum depression. The etiology of postpartum depression remains elusive. Etiology refers to the interaction of social, biological, psychological, and environmental factors Brummelte [12]; Yang et al. [13]. Studies have suggested that social factors play a significant role in the development of postpartum depression Zhang et al. [14]. Given the unique cultures and customs of Chinese society, these factors may be distinctly different in the Chinese population. However, in recent years, very few studies examined the social factors of postpartum depression among Chinese women. This literature review aims to address this gap by investigating the social factors of postpartum depression among Chinese women by reviewing the latest research to provide guidance for the identification, prevention, and treatment of postpartum depression.

\section{METHODS}

\section{Search Strategy}

Literature searches were conducted using multiple databases, including MEDLINE/PubMed, Google Scholar, and China National Knowledge Infrastructure. Search terms used include postpartum depression, or postnatal depression, Chinese women, social factors, social support, socio-cultural factors, doing the month or zuo yuezi, socio-economic factors, socio-demographic factors. Articles were excluded if the methodology was not clear or if they were of poor quality. After filtering for articles in English and Chinese with human participants published from January 12005 , to December 31 2020, 65 full text studies were identified, 3 clinical trials did not include the social factors of postpartum depression.

\section{Inclusion Criteria}

Studies were included if they: using a validated diagnosis of postpartum depression; participants were identified as Chinese women; reported data including prevalence of postpartum depression and social factors of postpartum depression.

\section{RESULTS}

\section{Search result}

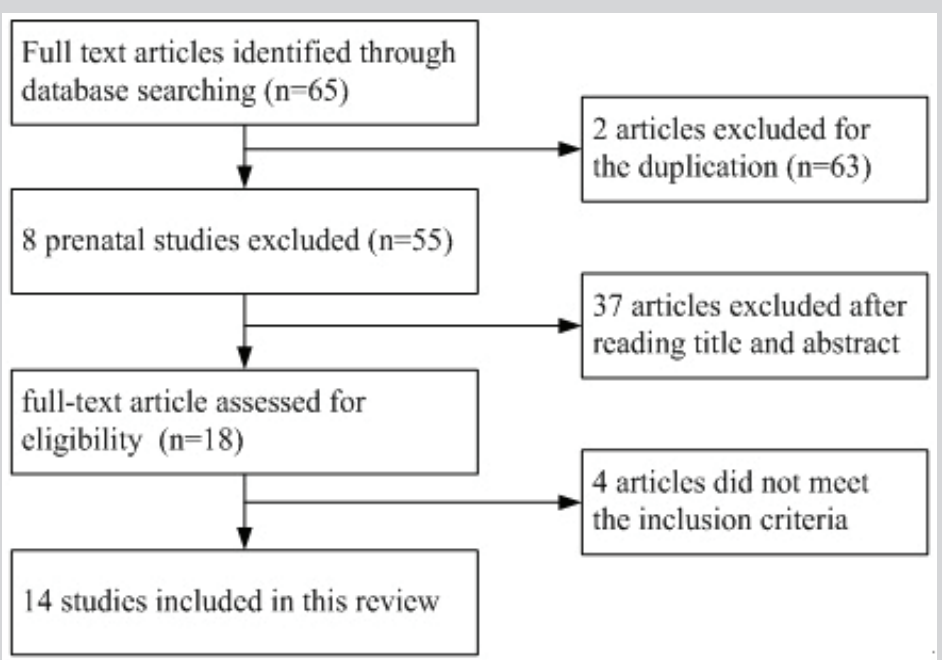

Figure 1: Flow chart of the study selection process.

65 studies were identified through dataset searching, 14 studies were included in this study after excluding 51 studies that did not meet the inclusion criteria. The selection process was shown in Figure 1. The characteristics of 14 included studies were presented in Table 1. Social factors refer to socio-cultural, socio- demographic factors and social support in this study.

\section{The Socio-Cultural Factors of Postpartum Depression in China}

\section{Son preference in Chinese culture}

China has some unique socio-cultural factors closely linked to postpartum depression; one of these is the strong preference for male infants in Chinese society. There is a deeply rooted belief that boys will bring more income to the family and society, and they will continue the family lineage. This was especially true since the implementation of the one-child policy in China in the late 1970s Chen et al. [15]; Wu et al. [16]; Hua et al. [17] carried out a prospective cohort study of 1,730 women in Shanghai, one of the biggest cities in China, to explore the relationship between infant gender and the wellbeing of postpartum women under the birth control policy. The General Wellbeing Schedule, a validated scale in America and Asian countries, was used at baseline (at 29-30 weeks gestation) and follow-up investigation (1-2 weeks after childbirth) to evaluate maternal wellbeing. It was found that, Chinese women with a female infant were at a higher risk of postpartum depression than women with a male infant. However, this association was moderated by the two-childbirth control policy. 
Table 1: Characteristics of included studies of social factors of postpartum depression.

\begin{tabular}{|c|c|c|c|}
\hline Sample Size & Measurement Timing & Risk Factors & Result \\
\hline 1,442 & EPDS 6 months postpartum & $\begin{array}{l}\text { Social-demographic factors between } \\
\text { Han-majority and Kazak-minority }\end{array}$ & $\begin{array}{l}\text { Kazak had a higher incidence of PPD (16.1\%) } \\
\text { than Han }(14.6 \%) \text {. Mother-in-law as a caregiver is } \\
\text { positively associated with PPD among Han, but not } \\
\text { with Kazak }\end{array}$ \\
\hline 190 & $\begin{array}{l}\text { EPDS, Structured questionnaire } \\
\text { One year postpartum }\end{array}$ & $\begin{array}{l}\text { Adherence to the cultural "doing the } \\
\text { months" practices }\end{array}$ & $\begin{array}{l}\text { Adherence to the "doing the month" practice was } \\
\text { negatively related to the PPD for the Chinese and } \\
\text { Vietnamese immigrant women }\end{array}$ \\
\hline 1,823 & EPDS 4 weeks postpartum & $\begin{array}{l}\text { education, fetus gender, housing } \\
\text { condition, income }\end{array}$ & $\begin{array}{c}\text { The PPD incidence was } 27.37 \% \text {, female fetus, small } \\
\text { area of housing were main contributors }\end{array}$ \\
\hline 2,615 & EPDS 42 days postpartum & $\begin{array}{l}\text { Traditional "doing the month" } \\
\text { practices }\end{array}$ & $\begin{array}{l}\text { Confinement practices and refreshing the indoor } \\
\text { air decreased the risk of PPD, sleep deprivation } \\
\text { increased the risk of PPD }\end{array}$ \\
\hline 1,730 & $\begin{array}{l}\text { GWBS, } 29-30 \text { weeks gestation } \\
\text { and } 1-2 \text { weeks postpartum }\end{array}$ & Infant's gender, birth control policy & $\begin{array}{l}\text { Women giving birth to male infants were positively } \\
\text { associated with the postpartum wellbeing, and it } \\
\text { can be moderated by the birth control policy }\end{array}$ \\
\hline 1,200 & EPDS 2-5 days postpartum & $\begin{array}{l}\text { Concern for face and willingness to } \\
\text { help seeking }\end{array}$ & $34.4 \%$ had early postnatal depressive symptoms, \\
\hline 2,365 & $\begin{array}{l}\text { EPDS Second trimester, third } \\
\text { trimester and postpartum }\end{array}$ & $\begin{array}{l}\text { Demographic, socio-economic and } \\
\text { social support }\end{array}$ & $\begin{array}{l}\text { Social support moderated the relationship between } \\
\text { PPD and mother-in-law conflicts }\end{array}$ \\
\hline 385 & $\begin{array}{c}\text { EPDS } 36 \text { weeks of gestation and } 6 \\
\text { weeks of delivery }\end{array}$ & Psychosocial variables & $\begin{array}{l}\text { 19.8 had PPD, perceived stress and childcare stress } \\
\text { were the risk factors of PPD }\end{array}$ \\
\hline 240 & $\begin{array}{l}\text { EPDS, PSSS Late pregnancy and } 4 \\
\text { weeks postpartum }\end{array}$ & $\begin{array}{l}\text { Perceived social support at three } \\
\text { different time point }\end{array}$ & $\begin{array}{l}\text { Perceived social support changed over the perinatal } \\
\text { and postnatal period, social support was an } \\
\text { important buffer against depression. }\end{array}$ \\
\hline 556 & $\begin{array}{l}\text { Two self-administered } \\
\text { questionnaires } 36-40 \text { weeks of } \\
\text { gestation }\end{array}$ & Socio-demographic factors & $\begin{array}{c}17.3 \% \text { had PPD, } 45.1 \% \text { women without PPD and } \\
58.9 \% \text { women with symptoms had parents-in-law } \\
\text { as caregiver. Intensive involvement of parents- } \\
\text { in-law, discriminating against a female baby, } \\
\text { inadequate support from husband are risk factors } \\
\text { for PPD }\end{array}$ \\
\hline 342 & EPDS 6-8 weeks postpartum & $\begin{array}{l}\text { Socio-demographic factors, zuoyuezi } \\
\text { and social support }\end{array}$ & $\begin{array}{l}\text { The prevalence of PPD was } 15.5 \% \text {, PPD was } \\
\text { associated with the lower income, low spousal } \\
\text { involvement and mother-in-law as caregiver }\end{array}$ \\
\hline 512 & EPDS 6 weeks postpartum & $\begin{array}{l}\text { Demographic and socio-cultural } \\
\text { factors among mainland China and } \\
\text { Taiwan women }\end{array}$ & $\begin{array}{c}\text { Social support, self-esteem were the significant } \\
\text { predictors of PPD in Taiwan; Education, social } \\
\text { support and self-esteem were significant predictors } \\
\text { of PPD in mainland China }\end{array}$ \\
\hline 534 & $\begin{array}{l}\text { SSRS, EPDS } 30-32 \text { weeks } \\
\text { of gestation and } 2 \text { weeks } \\
\text { postpartum }\end{array}$ & $\begin{array}{l}\text { Socio-demographic factors, social } \\
\text { support }\end{array}$ & $\begin{array}{c}\text { 19.29\% had PPD, women giving birth to female } \\
\text { infants had lower prenatal and postnatal social } \\
\text { support and higher rates of PPD }\end{array}$ \\
\hline 1,230 & EPDS 6 weeks postpartum & $\begin{array}{l}\text { inadequate family income, poor social } \\
\text { support, and marital relationships. }\end{array}$ & $\begin{array}{l}\text { 34.0\% migrant women had PPD, insufficient family } \\
\text { income, lower social support and poor marital } \\
\text { relationship were significantly associated with PPD }\end{array}$ \\
\hline
\end{tabular}

Abbreviations: PPD: Postpartum Depression; EPDS: Edinburgh Postnatal Depression Scale; GWBS: General Well-Being Schedule; SSRS: Social Support Rating Scale; PSSS: Perceived Social Support Scale.

Similarly, Xie et al. [18] conducted a prospective cohort study of 534 Chinese women to examine the association between social support and postpartum depression. Social support level was measured with the Social Support Rating Scale (SSRS), Edinburgh Postnatal Depression Scale (EPDS); Cox [19] was used to assess the postpartum depression and the demographic data was collected through face-to-face interviews. Results revealed that the women giving birth to a female infant had a much lower postpartum social support score and had a significantly higher risk of postpartum depression. A recent meta-analysis also indicates that women in Chinese society giving birth to a female infant are associated with a higher risk of developing postpartum depression than those giving birth to a male infant Ye et al. [20]. This finding is consistent with the study by Goyal et al. [21] in India. The strong preference for boys in Chinese culture is decreasing with rapid economic development, the two-child policy, and the gender equality promotion campaigns launched by the Chinese authorities Zhou et al. [22]. By contrast, the study by Lagerberg [23] found that, in Sweden, significantly more mothers of male infants experience postpartum depression symptoms than that mother of female infants.

\section{Cultural childbirth practice of "doing the month"}

As part of Chinese culture, the traditional postpartum custom of "doing the month" or zuo yuezi has been practiced for 2,000 years. It usually starts immediately after delivery and continues for a full month Pillsbury [24]. According to traditional Chinese 
medicine principles, childbirth contributes to an imbalance of yin and yang within the female body Liu et al. [25]. Close female family members, usually the mother or mother-in-law, will care for the postpartum woman so that the body harmony can be regained Holroyd et al. [26]. Also, during the first postpartum month, to protect postpartum women from illnesses, including postpartum depression, bathing, cold food, and going outside should be avoided Lee et al. [27].

The cultural postpartum ritual of "doing the month" provides traditional family support and contributes to the low rates of postpartum depression Ding et al. [28]. A cross-sectional study of Chen et al. [29] recruited 190 Chinese and Vietnamese immigrant mothers in Taiwan to examine the association between adherence to the "doing the month" practice and postpartum depression, risk factors were collected by using a structured questionnaire, depressive symptoms were measured using the Edinburgh Postnatal Depression Scale (EPDS), finding that adhering the cultural "doing the month" practices was negatively linked to the postpartum depression for both Chinese and Vietnamese immigrant women.

On the other hand, a growing number of studies suggest that adhering to these traditions is paradoxical in a modern healthy lifestyle, even leading to an increased risk of postpartum problems Wong [30]; Ding et al. [31]; Liu et al. [32] recruited 2,615 Shanghai postpartum women to examine the association between the traditional Chinese practice of "doing the month" and the risk of postpartum depression. The Edinburgh Postnatal Depression Scale scores were used on the 43rd day after delivery to collect the data. It was found that women who went outside their homes during the first month and had relatively less sleep been more likely to suffer from postpartum depression, and women who kept the windows open exhibited decreased risks of postpartum depression. Leung et al. [33] investigated the risk factors of postpartum depression among 385 Hong Kong women and found that perceived stress and childcare stress were the main psychosocial variables.

\section{Conflicts with mothers-in-law}

In Chinese society, it is quite common for mothers-in-law to care for postpartum women and their infants. Conflicts happen easily and could, to a certain extent, affect the social support provided in Chinese households. New mothers often have a different lifestyle and way of caring for their infant, leading to clashes with the traditional expectations of their mothers or mothers-in-law. Li et al. [34] conducted a cross-sectional study of 556 Chinese women to investigate the Chinese social and clinical factors causing postpartum depression. Two self-administered questionnaires (including the participants' age, body weight, educational level, occupation, family income, husband/attitude of parents-in-law toward a female baby, self-evaluation of husband's support, and so on) were performed by face-to-face interviews or follow-up telephone calls to collect the social factors of participants. The Edinburgh Postnatal Depression Scale was used at the 2 nd to the 4 th postnatal months to screen for postpartum depression symptoms. The results show that intense involvement of parents-in-law, discrimination against a female baby, and lack of support from the husband are the three main contributors to postpartum depression. This finding is consistent with the study by Deng et al. [35], investigating the risk factors of 1,823 postpartum women in Tangxia Community, Guangzhou, China, finding that an intense relationship between mother-in-law and daughter-in-law, female infant and cesarean delivery are risk factors for postpartum depression.

\section{Social stigma of mental illness in Chinese culture}

Mental illnesses, including postpartum depression, are stigmatized in Chinese society. Mental illness is considered to indicate an inability to solve problems or out-of-control behavior, bringing shame to the individual and the family Kramer et al. [36]. A substantial proportion of postpartum women are reluctant to disclose their condition. They prefer to deny their symptoms because postpartum depression patients face enormous life challenges. They are unable to bear the social stigma, embarrassment, and guilt Horowitz 2006. In some areas of China, it is not acceptable to discuss or acknowledge that someone in the family has depressive symptoms. This situation further prevents postpartum depressive women from having timely diagnosis and treatment Hung [37]. A cross-sectional comparative study by Lau [38] investigated the socio-cultural factors of 1,200 postpartum women in Hong Kong who had given birth 2-5 days previously. The results indicated that the traditional Chinese value of "face" and the inherent stigma of mental illness led women to deny having postnatal depressive problems and to refrain from seeking help.

\section{Socio-Demographic Factors}

\section{Socio-economic factors and education}

International studies have shown that women with low socioeconomic status Bennett et al. [39]; Chandran et al. [40] and lower education level Inandi et al. [41] are known to have a particularly high risk of prenatal and postpartum depression. According to a study conducted by Vericker et al. [42], about $41 \%$ of 9 -monthold babies in the United States have a mother with some form of depression; the proportion of people living below the poverty line has risen to $55 \%$.

China is experiencing rapid economic development and urbanization. Rural to urban migration is a major source of China's growth, and living conditions, one of the main concerns among migrants, have negative effects on women's physical and mental health Hu et al. [43]; Xiong [6] investigated 1,230 immigrant women in Guangzhou city in south China and found that the development of postpartum depression is associated with social factors, including living in Guangzhou for less than two years, insufficient family income, poor social support, and problematic marital relationships. Chen et al. [44] conducted a systematic review of risk factors of postpartum depression in Chinese immigrant women. They found that low social support and acculturation are negatively associated with postpartum depression. Wang et al. [45] recruited 512 mothers in Taiwan and Fuzhou city in mainland China. Five selfreport instruments were used to collect data. Results reveal that social supports are significant predictors of postpartum depression for women living in Taiwan, and education and social support were the significant predictors of postpartum depression for women living in Fuzhou.

\section{Diverse ethnicities in Chinese society}

China is a multi-ethnic nation, with 55 ethnic minority groups and a Han majority. However, very limited studies are targeted at postpartum women in Chinese minorities. Chen et al. [46] explored the ethnic differences of associated socio-demographic factors for postpartum depression between Han-majority and Kazak-minority women in a less-developed region in northwestern China. A total of 1,442 women were invited to participate in this study that found that having mothers-in-law as caregivers was positively associated 
with postpartum depression among Han but not among Kazak women. Common risk factors (previous pregnancy loss, giving birth to a female infant) existed among both ethnic groups. The study of Wan et al. [47] investigated 343 Chinese women in Beijing with 6-8 postpartum and found that women with mother-in-law as caregiver had a two times PPD prevalence than others. International studies had also indicated that ethnic inequalities are closely linked to the prevalence of and risk factors for postpartum depression Shwartz et al. [48].

\section{Social support for postpartum depression}

Social support is a comprehensive term that is defined as different aspects of social relationships, emphasizing the resources provided by others Cohen [49]. Social support consists of support from various sources such as family members, colleagues, friends, neighbors, professionals, and organizations Xiao [50]. Dealing with various new maternal tasks every day is a considerable challenge for postpartum women Walker [51]. Social support helps to alleviate the overwhelming responsibilities of childcare and housework for new mothers Hung [37]. More importantly, in their transition from wives to mothers, social support can help women develop self-confidence and self-esteem by providing understanding and appreciation Dennis [52].

\section{Family support for Chinese postpartum women}

In the family-centered Chinese society, family support is likely to be one of the most important components of social support. A prospective cohort study involving 534 pregnant women found that lack of postnatal family support, especially support from the husband, is an important risk factor for postpartum depression Xie et al. [53]. Research demonstrates that new mothers will have a high risk of postpartum problems if the emotional support from their husbands is absent. This also leads to a poor marital relationship, and it is a major risk factor for postpartum depression Tang et al. [54].

Chinese husbands are generally less likely to provide support for their wives in the form of baby care, due to the Chinese masculine culture and traditional rituals Hofstede et al. [55]. Furthermore, in traditional Chinese culture, sons should respect their parents' opinions, which could result in reduced support for their wives Xie et al. [56]. Reduced support from a husband would lead to a high risk of postpartum depression for a wife. In a qualitative study involving interviewing 35 Hong Kong Chinese women, perceived lack of support from husbands and families was identified as a possible contribution to postpartum depression Chan [57]. This finding is also supported by researchers from western countries. For example, Stapleton et al. [58] mentioned that partner support can predict postpartum depression at eight weeks, and that women who receive emotional support from their intimate partner tend to have a lower level of emotional disorder at 6-8 weeks postpartum.

\section{The association between social support and postpartum depression}

Social support helps prevent, identify, and treat postpartum depression Howell et al. [59]. Previous studies in China have indicated that depressive symptoms may result from poor social support because lack of social support will cause negative emotions and experiences Leahy [60].

Faleschini et al. [9] conducted a prospective cohort study in China to examine the relationship between prenatal and postnatal social support and postpartum depression. The results showed that women with lower social support had a higher risk of postpartum depression. In addition, postpartum social support predicts postpartum depression risk more effectively than prenatal support. Li et al. [61] recruited 240 pregnant women. They investigated the changes in the prevalence of depression and the level of social support at three different time points: late pregnancy, one week postpartum, and four weeks postpartum. They examined the relationship between depression and social support at these points and found that women with higher social support had less likelihood of developing depression.

Lau [62] investigated 2,365 Hong Kong women. The Interpersonal Support Evaluation List was used to measure the functional aspects of the perceived availability of social support. The women were identified as having depressive symptoms according to the Edinburgh Postnatal Depression Scale. The results show that social support has a moderating effect on the relationship between antenatal depressive symptoms and marital and motherin-law conflicts and on postnatal depressive symptoms in conflicts with the father-in-law. Social support also plays a mediating role in the relationship between marital and mother-in-law conflicts and postnatal depressive symptoms [63].

\section{Implication and Limitations of this Study}

Further research for social factors of postpartum depression among Chinese women is needed to clarify this result. There are also some limitations to this study. First, the methodologies of the included studies are diverse. Therefore, there is a possibility of low-quality evidence. Second, very few studies explore postpartum depression among women from Chinese minorities and women living in rural areas. Third, in some included studies, depressive symptoms were measured by self-rated depression scales, which could affect the reliability of results. Finally, some studies only assessed perceived social support but not actual social support. However, these parameters were usually correlated [64].

\section{CONCLUSION}

This article provides a detailed review of the social factors of postpartum depression among Chinese women. On the one hand, this study reveals that a variety of unique socio-cultural factors are associated with postpartum depression. The factors include son preference, the traditional "doing the month" custom, and the conflict between postpartum women and mothers-in-law in Chinese culture. On the other hand, socio-demographic factors, such as income, education, and ethnicity, are also considered risk factors for postpartum depression in China. Finally, social support positively influences Chinese women's postpartum experience, especially family support. These findings contribute to the prevention, detection, and treatment of postpartum depression among Chinese women. Also, the unique social factors of postpartum depression should be considered by Chinese authorities in health planning and policymaking. There is a need to bridge the gap between traditional practices and current evidence about the social factors of postpartum depression so that health care workers can provide effective, evidence-based, culturally competent postpartum care.

\section{REFERENCES}

1. Hahn HJ, Cornwell HT, Anaya I (2017) Economic and health predictors of national postpartum depression prevalence: a systematic review, metaanalysis, and meta-regression of 291 studies from 56 countries. Front Psychiatry 8: 248 . 
2. World Health Organization (2013) Mental health and substance use.

3. Wisner KL, Sit DKY, Shea MC, David MR, Rebecca AZ, et al. (2013) Onset timing, thoughts of self-harm, and diagnoses in postpartum women with screen-positive depression findings. JAMA Psychiatry 70(5): 490-498.

4. Schardosim JM, Heldt E (2011) Postpartum depression screening scales: a systematic review. Revista gaúcha de enfermagem 32(1): 159-166.

5. Zhang Y, Zhang WY, Zhou L (2013) Discussion on the related risk factors of postpartum depression. Chinese J Laboratory Diagnosis 17(1): 85-87.

6. Xiong R, Deng A (2020) Prevalence and associated factors of postpartum depression among immigrant women in Guangzhou, China. BMC Pregnancy and Childbirth 20(1): 247.

7. Ludermir AB, Lewis G, Valongueiro SA (2010) Violence against women by their intimate partner during pregnancy and postnatal depression: a prospective cohort study. The Lancet (British edition) 376: 903-910.

8. Closa MR, Gispert LLM, Canals J, Luque V, Zaragoza MJ, et al. (2017) The effects of Postpartum depression and current mental health problems of the mother on child behaviour at eight years. Maternal \& Child Health J 21(7): 1563-1572.

9. Faleschini S, Rifas SSL, Tiemeier H, Oken E, Hivert M, et al. (2019) Associations of prenatal and postnatal maternal depressive symptoms with offspring cognition and behavior in Mid-Childhood: A prospective cohort study. Int J Environ Res Public Health 16: 1007.

10. Koutra K, Roumeliotaki T, Kyriklaki A, Mariza K, Katerina S, et al. (2017) Maternal depression and personality traits in association with child neuropsychological and behavioral development in preschool years: Mother-child cohort (Rhea Study) in Crete, Greece. Journal of Affective Disorders 217(8): 89-98.

11. Saralee G, Daphna L, Ethel Sherry G (2018) The tip of the iceberg: postpartum suicidality in Israel. Israel J Health Policy Research 7(1): $1-12$.

12. Brummelte S, Galea LAM (2016) Postpartum depression: Etiology, treatment and consequences for maternal care. Hormones and behavior 77: $153-166$

13. Yang F, Gardner CO, Bigdeli T, Jingfang G, Zhen Z, et al. (2015) Clinical features of and risk factors for major depression with history of postpartum episodes in Han Chinese women: A retrospective study. J Affective disorders 183: 339-346.

14. Zhang Y, Zou S, Cao Y, Zhang Y (2012) Relationship between domestic violence and postnatal depression among pregnant Chinese women. International J Gynecology Obstetrics 116(1): 26-30.

15. Chen J, Xie Z, Liu H (2007) Son preference, use of maternal health care, and infant mortality in rural China, 1989-2000. Population studies 61: 161-183.

16. Wu Z, Viisainen K, Hemminki E (2006) Determinants of high sex ratio among newborns: A cohort study from rural anhui province, China Reproductive Health Matters 14(27): 172-180

17. Hua J, Zhu L, Du W, Du L, Luo T, et al. (2016) Infant's sex, birth control policy and postpartum well-being: a prospective cohort study in Shanghai, China. BMJ open 6(10): e012207-e012207.

18. Xie R, He G, Koszycki D, Walker M, Wen SW, et al. (2009) Fetal sex, social support, and postpartum depression. Canadian J Psychiatry 54(11) $750-756$.

19. Cox J (1987) Origins and development of the 10 item edinburgh depression scale. In: Perinatal psychiatry: use and misuse of the Edinburgh postnatal depression scale. Gaskell, London, pp. 115-124.

20. Ye Z, Wang L, Yang T, Li ZC, Tingting W, et al. (2020) Gender of infant and risk of postpartum depression: a meta-analysis based on cohort and case-control studies. J Maternal Fetal Neonatal Med: 1-10.

21. Goyal K, Purbiya P, Lal SN, Kaur J, Anthwal P, et al. (2017) Correlation of infant gender with postpartum maternal and paternal depression and exclusive breastfeeding rates. Breastfeed Med 12: 279-282.
22. Zhou C, Zhou C, Wang XL, Therese H (2012) Son preference and sexselective abortion in China: informing policy options. Int J Public Health 57(3): 459-465.

23. Lagerberg D, Magnusson M (2012) Infant gender and postpartum sadness in the light of region of birth and some other factors: a contribution to the knowledge of postpartum depression. Archives of women's mental health 15(2): 121-130.

24. Pillsbury BLK (1978) "Doing the month": Confinementand convalescence of Chinese women after childbirth. Social science \& medicine. Part B Medical anthropology 12: 11-22.

25. Liu YQ Petrini M, Maloni JA (2015) “Doing the month": Postpartum practices in Chinese women. Nursing \& health sciences 17(1): 5-14.

26. Holroyd E, Twinn S, Yim IW (2004) Exploring Chinese women's cultural beliefs and behaviors regarding the practice of "Doing the Month". Women \& health 40(3): 109-123.

27. Lee S, Yang S, Yang Y (2013) Doing-in-month ritual among Chinese and Chinese American. J Cultural Diversity 20: 94.

28. Ding G, Tian Y, Yu J, Vinturache A (2018) Cultural postpartum practices of 'doing the month' in China. Perspectives public health 138: 147-149.

29. Chen T, Tai C, Wu T, Chiang C, Chien L, et al. (2012) Postpartum cultural practices are negatively associated with depressive symptoms among Chinese and Vietnamese immigrant mothers married to Taiwanese men. Women \& health 52: 536-552.

30. Wong J, Fisher J (2009) The role of traditional confinement practices in determining postpartum depression in women in Chinese cultures: A systematic review of the English language evidence. J Affective Disorders 116(3): 161-169.

31. Ding G, Niu L, Vinturache A, Jun Z, Min L, et al. (2020) "Doing the month" and postpartum depression among Chinese women: A Shanghai prospective cohort study. Women and birth: J Australian College of Midwives 33: e151-e158.

32. Liu YQ Maloni JA, Petrini MA (2014) Effect of postpartum practices of doing the month on Chinese women's physical and psychological health. Biol Res Nurs 16: 55-63.

33. Leung SSK, Martinson IM, Arthur D (2005) Postpartum depression and related psychosocial variables in Hong Kong Chinese women: Findings from a prospective study. Research in nursing \& health 28(1): 27-38.

34. Li Q Yang S, Xie M, Xiaoming W, Liping H, et al. (2020) Impact of some social and clinical factors on the development of postpartum depression in Chinese women. BMC Pregnancy and Childbirth 20: 226.

35. Deng A, Xiong R, Jiang T, Luo Y, Chen W, et al. (2014) Prevalence and risk factors of postpartum depression in a population-based sample of women in Tangxia Community, Guangzhou. Asian Pacific J of tropical medicine 7: 244-249.

36. Kramer EJ, Kwong K, Lee E, Chung H (2002) Cultural factors influencing the mental health of Asian Americans. West J Med 176(4): 227-231.

37. Hung C, Chung H (2001) The effects of postpartum stress and social support on postpartum women's health status. J Adv Nurs 36: 676-684.

38. Lau Y, Fu KWD (2008) Are concern for face and willingness to seek help correlated to early postnatal depressive symptoms among Hong Kong Chinese women? A cross-sectional questionnaire surveys. International J nursing studies 45: 51-64.

39. Bennett HA, Einarson A, Taddio A, Koren G, Einarson TR, et al. (2004) Prevalence of depression during pregnancy: systematic review. Obstetrics and gynecology 103: 698-709.

40. Chandran M, Tharyan P, Muliyil J, Abraham S (2002) Post-partum depression in a cohort of women from a rural area of Tamil Nadu, India. Incidence and risk factors. British J psychiatry 181: 499-504.

41. Inandi T, Elci OC, Ozturk A, Egri M, Polat A, et al. (2002) Risk factors for depression in postnatal first year, in eastern Turkey. International J Epidemiology 31: 1201-1207. 
42. Vericker J, Macomber 0 (2010) Golden infants of depressed mothers living in poverty: opportunities to identify and serve. The Urban Institute.

43. Hu F, Xu Z, Chen Y (2011) Circular migration, or permanent stay? Evidence from China's rural-urban migration. China Economic Review 22: 64-74.

44. Chen J, Cross WM, Plummer V, Lam L, Tang S, et al. (2019) A systematic review of prevalence and risk factors of postpartum depression in Chinese immigrant women. Women and birth: J Australian College of Midwives 32: 487-492.

45. Wang S, Jiang X, Jan W, Chen C (2003) A comparative study of postnatal depression and its predictors in Taiwan and mainland China. American Obstet Gynecol 189: 1407-1412.

46. Chen L, Ding L, Qi M, Jiang C, Mao X, et al. (2018) Incidence of and social-demographic and obstetric factors associated with postpartum depression: differences among ethnic Han and Kazak women of Northwestern China. Peer J 6: e4335-e4335.

47. Wan EY, Moyer CA, Harlow SD, Fan Z, Jie Y, et al. (2009) Postpartum depression and traditional postpartum care in China: Role of Zuoyuezi. Int J Gynecol Obstet 104: 209-213

48. Shwartz N, Shoahm VI, Daoud N (2018) Postpartum depression among Arab and Jewish women in Israel: Ethnic inequalities and risk factors. Midwifery 70: 54-63.

49. Cohen S, Wills TA (1985) Stress, Social Support and the Buffering Hypothesis. Psycho bull 98(2): 310-357.

50. Xiao S (1999) Social support rating scale. In: Wang X (Edn) Mental health scale, Chinese Mental Health Publishing House, Beijing, China, pp: 35-45.

51. Walker LO, Wilging S (2000) Rediscovering the "M" in "MCH": Maternal health promotion after childbirth. J Obstet Gynecol Neonatal Nurs 29(3): 229-236.

52. Dennis C, Chung LL (2006) Postpartum depression help-seeking barriers and maternal treatment references: A Qualitative Systematic Review. Birth 33: 323-331.
53. Xie R, Yang J, Liao S, Xie H, Walker M, et al. (2010) Prenatal family support, postnatal family support and postpartum depression. Australian \& New Zealand J Obstetrics \& Gynaecology 50(4): 340-345.

54. Tang L, Zhu R, Zhang X (2016) Postpartum depression and social support in China: A Cultural perspective. J Health Communication 21(9): 1055-1061.

55. Hofstede GH, Hofstede GJ, Minkov M (2010) Cultures and organizations: Software of the mind: intercultural cooperation and its importance for survival. $3^{\text {rd }}$ edn, Mc Graw-Hill, New York, USA

56. Xie RH, He G, Koszycki D, Walker M, Wen SW, et al. (2009) Prenatal social support, postnatal social support, and postpartum depression. Annals of Epidemiology 19(9): 637-643.

57. Chan S, Levy V (2004) Postnatal depression: a qualitative study of the experiences of a group of Hong Kong Chinese women. J of Clinical Nursing 13(1): 120-123.

58. Stapleton LRT, Schetter CD, Westling E, Christine R, Laura MG, et al. (2012) Perceived partner support in pregnancy predicts lower maternal and infant distress. J Family Psychology 26: 453-463.

59. Howell EA, Mora P, Leventhal H (2006) Correlates of early postpartum depressive symptoms. Maternal and Child Health J 10: 149-157.

60. Leahy Warren P (2005) First-time mothers: social support and confidence in infant care. J advanced nursing 50(5): 479-488.

61. Li Y, Long Z, Cao D, Cao F (2017) Social support and depression across the perinatal period: A longitudinal study. J Clinical Nursing 26: 27762783.

62. Lau Y, Keung WDF (2008) The Role of social support in helping Chinese women with perinatal depressive symptoms cope with family conflict. Journal of obstetric, gynecologic, and neonatal nursing 37: 556-571.

63. American Psychiatric Association (2013) Diagnostic and statistical manual of mental disorders (DSM-V) $5^{\text {th }}$ edn, Arlington, USA.

64. Su D, Wu X, Zhang Y, Hui PL, Wei LW, et al. (2012) Depression and social support between China' rural and urban empty-nest elderly. Archives of gerontology and geriatrics 55(3): 564-569. 